\title{
Abnormal uterine bleeding: hysterectomy versus resection
}

\author{
Dender Soares Santiago ${ }^{1}$ \\ Jordan Barros da Silva ${ }^{2}$ \\ Renato Martins Pereira ${ }^{3}$ \\ Cintia do Couto Mascarenhas ${ }^{1}$
}

\begin{abstract}
1. Escola de Saúde e Medicina, Curso de Medicina, Universidade Católica de Brasília, Brasília, DF, Brasil 2. Laboratório de Micologia do Núcleo de Parasitologia e Micologia (NPM) do Laboratório Central de Saúde Pública do Distrito Federal (LACEN-DF), Brasília, DF, Brasil 3. La Femme Ginecologia de Excelência Ltda, Brasília, DF, Brasil
\end{abstract}

http://dx.doi.org/10.1590/1806-9282.66.12.1731

KEYWORDS: Uterine Hemorrhage. Menorrhagia. Hysterectomy. Endometrial Ablation Techniques. Endometrium/surgery. PALAVRAS-ChaVE: Hemorragia Uterina. Menorragia. Histerectomia. Técnicas de Ablação Endometrial. Endométrio/cirurgia.

\section{INTRODUCTION}

Abnormal uterine bleeding (AUB) is one of the most common clinical conditions in gynecology. It may compromise alterations in menstrual cycle duration, frequency, and regularity of flow ${ }^{1}$. It is estimated that about $10 \%$ to $30 \%$ of women will be affected by AUB during their lifetime, and more than 10 million American women are affected by it each year ${ }^{2,3}$. It influences the quality of life affecting physical, emotional and social well-being ${ }^{3,4}$. Menorrhagia mostly affects women of childbearing age and is more common in menarche and perimenopause, often causing excessive uterine bleeding, fatigue, and difficulty performing daily activities ${ }^{5,6}$.

The diagnose of AUB is a big challenge in medical practice, requiring thorough clinical analysis and careful observation of inconclusive parameters. Concomitantly, there are suggestive diagnosis aspects that should be ruled out upon examination such as anemia, hypothyroidism, polycystic ovary syndrome, coagulation disorders, and clonal diseases such as endometrial cancer, colon, and thyroid tumours ${ }^{3}$. To reduce the difficulties of the diagnostic, a classification system for menorrhagia was created to be used worldwide, the Palm-Coein classification system ${ }^{5}$. Developed by the International Federation of Obstetrics and Gynaecology (FIGO), its letters represent an acronym with the possible problems associated with AUB (polyps, adenomyosis, leiomyomas, malignancy, ovulatory dysfunction, endometrial disorders, iatrogenic factor $)^{5,7}$.

Initially, the treatment for abnormal uterine bleeding consists of clinical attention using drugs or a levonorgestrel-releasing intrauterine device. The surgical procedure is best suited for women who have no 
intention to become pregnant and is usually preceded by an unsuccessful clinical treatment, normally is performed using hysterectomy or endometrial ablation resection techniques ${ }^{8}$.

The surgery in which the uterus is totally or partially removed, hysterectomy, can be done by abdominal, vaginal, laparoscopic, or robot-assisted laparoscopic surgery, and abdominal hysterectomy is the most invasive approach ${ }^{8}$. Endometrial ablation, in turn, is a surgical method aimed at reducing uterine bleeding by removing a portion of the endometrial tissue layer. Among the ablation possibilities are the hysteroscopic or $1^{\text {st }}$ generation ablation techniques, which are more suitable for abnormal uterine cavities, leiomyomas, or polyps. This surgical class is characterized by endometrial resection that uses electrosurgical or laser tools. On the other hand, there is non-hysteroscopic or $2^{\text {nd }}$ generation ablation that is recommended for benign bleeding. The treatments for those cases include radiofrequency, hot fluid, cryotherapy, microwave, and laser or thermal balloon ablation techniques ${ }^{9,10}$. In general, newer endometrial ablation techniques are less invasive and are alternatives to hysteroscopic techniques.

There are few retrospective clinical studies or randomized controlled trials comparing hysterectomy and endometrial ablation techniques. Thus, current analyses are inconclusive as to which treatment options are the best. However, it is inferred that both techniques are effective in treating dysfunctional uterine bleeding, thus each clinical context must be considered.

\section{METHODS}

A literature review covering publications from 2007 to 2018 was conducted to select controlled clinical trials or retrospective studies that compared hysterectomy and resection with endometrial ablation techniques used to treat abnormal uterine bleeding (AUB). We searched the PubMed - NCBI, SciELO, BIREME, Cochrane, LILACS and MEDLINE databases using the words "abnormal uterine bleeding", "hysterectomy", "resection versus hysterectomy", "endometrial ablation and abnormal uterine bleeding", "endometrial", "ablation and hysterectomy", "endometrial ablation versus hysterectomy", "heavy menstrual bleeding”, "dysfunctional uterine bleeding”, "heavy menstrual bleeding”, "menorrhagia”, "laparoscopy" and "endometrial resection and ablation" as well as their respective translations in Portuguese databases.
Case studies, reviews, articles that compared surgical techniques with pharmacological treatments along with book chapters, theses, dissertations, and course completion papers were excluded from the study. We obtained 2,375 articles from the searches. Of the articles identified, only eight articles, five clinical trials (including multicentric studies) with recommendation grade $\mathrm{A}$ (levels $1 \mathrm{~A}$ and $1 \mathrm{~B}$ ), and three retrospective studies of recommendation grade $\mathrm{B}$ public databases (level 2C) according to the Oxford Centre classification for evidence-based medicine, fit the research. These articles deal with the positive and negative aspects of each surgical technique.

\section{RESULTS}

Regarding pain in the postoperative period, data from controlled studies vary; two controlled studies reported no significant differences in the 24-month period under review ${ }^{6,7}$. Two controlled studies state that pain was higher in patients undergoing hysterectomy than in those undergoing endometrial ablation $^{11,12}$. A randomized study that focused its analysis on the reoperation rate and quality of life did not analyze pain ${ }^{13}$. Articles with pain data analyzed post-surgery did not reach a consensus, yet they were favorable to endometrial ablation techniques.

Analyzing the quality of life after surgery, three controlled studies corroborate that both techniques are effective in treating abnormal uterine bleeding leading to significant improvements in patient well-being ${ }^{6,712}$. A randomized clinical trial concluded that thermal balloon endometrial ablation may replace vaginal hysterectomy for perimenopausal women with uterine leiomyomas by demonstrating good rates of response ${ }^{11}$. This study claimed all women undergoing amenorrhoeic endometrial ablation had reduced menstrual flow volume over a 12-month period. Another randomized trial concluded that laparoscopic supracervical hysterectomy resulted in a better quality of life when compared to hysteroscopic endometrial ablation $^{13}$. The clinical trial of Sesti et al. ${ }^{12}$, in which the SF-36 score was satisfactory in both groups, obtained a low result for emotional function in patients treated with laparoscopic supracervical hysterectomy, which was associated with the removal of the uterus. The Medical Outcomes Short-Form Health Survey (SF-36) was the most widely used instrument in controlled studies to assess the quality of life. In a randomized clinical trial, in addition to the SF-36, the abbreviated 
form of Health Metric's Health Shot form (SF-12v2), shortened from the SF-36, was used ${ }^{13}$. Finally, a randomized clinical trial used Symptom and Quality of Life (UFS-QOL) scores in its analysis before and after surgical procedures to conclude that there were improvements in the quality of life of patients treated for abnormal uterine bleeding when both techniques were compared ${ }^{11}$.

When comparing reoperation rates, studies show that endometrial ablation has considerable relapse rates $^{2,6,7,13-15}$. A randomized controlled trial focusing on reoperation rates and quality of life comparing laparoscopic supracervical hysterectomy with hysteroscopic endometrial ablation yielded a reoperation rate of $13.4 \%(12 / 89)$ after 24 months (12/89) for ablation and $1 \%(1 / 92)$ for those who underwent hysterectomy ${ }^{13}$. One randomized clinical trial had a $5 \%$ $(1 / 20)$ reoperation rate for patients who underwent thermal balloon ablation against none $(0 / 20)$ of those who underwent vaginal hysterectomy ${ }^{11}$. Another randomized clinical trial achieved a 24-month reoperation rate of 24.5\% (27/110) among women who underwent endometrial ablation, with the rate increasing to $31 \%$ $(34 / 110)$ at 60 months $^{6}$. In further studies and clinical analyses, another trial found reoperation rates of approximately $29 \%$ within 48 months for the group of women undergoing endometrial ablation ${ }^{7}$. A randomized clinical study did not report present data in this article $^{12}$. Three retrospective studies were also in line with data studies regarding findings that there are greater rates of reoperation for women undergoing ablation for $\mathrm{AUB}^{2,14,15}$.

Regarding bleeding, the literature concedes that hysterectomy is the definitive procedure to contain menorrhagia since this technique produces very high percentages of amenorrhea patients. Three randomized clinical trials have obtained significant results not only in solving the problem of excessive bleeding but also claim to have achieved considerable increases in hemoglobin levels for both surgical techniques ${ }^{7,11,12}$. One of the studies claims that in 24 months, $75 \%$ $(15 / 20)$ of women underwent a second-generation ablative technique with amenorrhea, compared to $20 \%$ $(4 / 20)$ who had mild bleeding and only $5 \%(1 / 20)$ of the patients in this group were reoperated for the same issue ${ }^{11}$. A multicentre, controlled study states that hysterectomy is more effective in stopping bleeding, but both groups performed well and were effective.

Analyzing postoperative complications, a randomized clinical trial reported nearly six times more infections and four times more adverse effects in the hysterectomy group ${ }^{6}$. One randomized clinical study reports greater adverse effects for hysterectomy, and three other trials did not find significant post-operative complications $\mathbf{6}^{6,11-14}$. Among the retrospective studies, two confirm data from the literature that hysterectomy is associated with an increased incidence of pelvic organ prolapse and promotes higher urinary incontinence rate $\mathrm{s}^{\mathbf{s}, 4,15}$. One study, a retrospective cohort study based on national Scottish hospital data from 1989 to 2006, points out that while there are few ablation complications, one should consider the fact that approximately a quarter of all cases require future surgery for $\mathrm{AUB}^{15}$. Finally, another retrospective cohort study showed that hysterectomy was associated with twice the chance (36\% versus 15\%; p $<0.001)$ of postoperative complications than endometrial ablation ${ }^{14}$.

A retrospective study assessing the risks of further surgery and gynecological cancer did not find any relevant incidence of postoperative cancer $(<1.6 \%)^{15}$. Another retrospective study compared the costs between second-generation endometrial ablation and hysterectomy in the period 2006-2010 from the US database and reported that the cost of hysterectomy is approximately twice as high as that of endometrial ablation $(\$ 12,147$ vs. $\$ 5,837)$ as well as demonstrates that the likelihood of short-term disability claims is four times higher (84\% vs 21\%; $p<0.001)^{14}$. Finally, a retrospective, longitudinal, observational study compared the clinical and economic benefits of radiofrequency ablation versus hysterectomy ${ }^{2}$. This study used the German health database as a source from January 2008 to September 2013 and concluded that the second-generation techniques save around $€ 1,844$ per case in the country and are less associated with adverse effects.

\section{DISCUSSION}

Abnormal uterine bleeding (AUB) is one of the most frequent menstrual cycle disorders worldwide, generally affecting women at the end of their fertile lives. It accounts for about one-third of disturbance cases and is characterized by excessive blood loss by volume, frequency, regularity, or duration of flow. AUB compromises the quality of life of many women, including their ability to perform daily activities, and the emotional, social, and physical aspects of their live ${ }^{\text {s1 }}$. Surgical techniques for treating AUB favorably reduce anxiety and depression that may have existed 
pre-surgery. However, excluding the first year after surgery hysterectomy is associated with a better quality of life and sexuality results when compared to endometrial resection ${ }^{16}$. There is a treatment based on the clinical history; however, diagnosis can be challenging due to the thorough clinical analysis required, and doctors are often only guided by patient reports ${ }^{3}$. In this context, hysteroscopy is an important diagnostic technique to assess the uterine cavity ${ }^{17}$. The possible visualization, biopsy, and confirmation of abnormal findings reveal the importance of this practice, which has several forceps that can be used ${ }^{17,18}$. Lin's biopsy forceps are flexible in the management of endometrial abnormalities, effectively helps hysteroscopy with both the biopsy and removal of uterine lesions ${ }^{17}$. Furthermore, the Vitale biopsy forceps were developed with the capacity to grab tissue and cut at the same time, thus making it useful in the field of ambulatorial hysteroscopy ${ }^{18}$. All of this technology involved in the diagnostic hysteroscopy technique provides greater accuracy for professionals when viewing and evaluating the interior of the uterus and finding the possible causes of the SUA ${ }^{17}$.

It is possible to choose one of the hysterectomy or resection techniques with endometrial ablation, and hysteroscopic techniques include abdominal, vaginal, laparoscopic, and robot-assisted laparoscopic hysterectomy $^{\mathbf{8}}$. Abdominal hysterectomy is achieved by an incision in the lower abdomen, and this technique is the most invasive. Vaginal hysterectomy is performed with no need for incisions and has a lower morbidity rate and recovery time than the abdominal option. Laparoscopic hysterectomy is less invasive, performed making fewer incisions or vaginally assisted by video accessories, and can also be performed by a robotic mechanism. Also, there is the resection with endometrial ablation, which is a technique used to dissect the endometrium layer, inducing tissue regeneration and the reduction of uterine bleeding. They are divided into $1^{\text {st }}$ generation hysteroscopic resections of the endometrium with electrosurgical tools, and as such more suitable for abnormal uterine cavities, leiomyomas or polyps, and non-hysteroscopic cavities ( $2^{\text {nd }}$ generation), recommended for women in whom bleeding is of benign cause. The $2^{\text {nd }}$ generation techniques are: radiofrequency ablation; thermal flask; hot fluid; cryotherapy; microwave; laser. Surgical options are usually considered after the patient is refractory to clinical treatment and should only be chosen after case analysis and detailing of uterine characteristics in order to determine the most appropriate technique.

The present article aims to compare hysterectomy and resection with endometrial ablation from a literature review. As a real clinical implication, those studies have shown that maybe the definitive technique for obtaining amenorrheic women is hysterectomy ${ }^{6,7,11-13}$. There was no consensus that one of the procedures was definitely the best but hysterectomy techniques were more resolutive and with fewer recurrences $^{2,6,7,11-15}$. Taking into account the viability of each method, both surgical classes can be used in clinical practice, taking into account the indications and contraindications when choosing.

There are several possible surgical techniques to be chosen, and the choice of the most appropriate method in each case must consider the age, the choice of the patient, the desire to become pregnant, anatomical changes, and the contraindications for the use of a surgical procedure. The patient's choice is influenced by variables such as recovery time, the fact that hysterectomy techniques are more invasive, the simplicity of endometrial ablation, and the achievement of amenorrhea when using hysterectomy. When comparing two classes of surgical techniques, it is notable that there are many variables to be paired and outlined in future studies in order to achieve greater accuracy on the best indication or even reach a consensus.

\section{CONCLUSION}

Even though all techniques have shown to be effective in treating this gynecological disorder, there is no consensus in the literature as to which one of the surgical techniques is definitively the best treatment option for abnormal uterine bleeding. In regard to improved quality of life, both are visibly beneficial. Endometrial ablation was related to a higher percentage of refractoriness as it preserves much of the uterine structure, whilst hysterectomy has been shown to be more effective in generating amenorrhea in patients than the others techniques. However, hysterectomy techniques were associated with higher rates of adverse effects such as pelvic organ prolapse and urinary incontinence. Among the hysteroscopic techniques, vaginal hysterectomy has been shown to cause greater adverse effects. There was no evidence of a relationship between gynecological cancer and treatments and there was no consensus on which technique promotes greater pain during the postoperative period. 


\section{REFERENCES}

1. Kalampokas E, McRobbie S, Payne F, Parkin DE. Long-term incidence of hysterectomy following endometrial resection or endometrial ablation for heavy menstrual bleeding. Int J Gynaecol Obstet. 2017;139(1):61-4.

2. Kessel S, Hucke J, Goergen C, Soeder R, Roemer T. Economic and clinical benefits of radiofrequency ablation versus hysterectomy in patients suffering from menorrhagia: a retrospective analysis with German health claims data. Expert Rev Med Devices. 2015;12(3):365-72.

3. Silva-Filho AL, Rocha ALL, Ferreira MCF Celani M, Lamaita R, Cândido $E B$, et al. Sangramento uterino anormal: proposta de abordagem do Grupo Heavy Menstrual Bleeding: Evidence-Based Learning for Best Practice (HELP). Femina. 2015;43(4):161-6.

4. Bhattacharya S, Middleton LI, Tsourapas A, Lee A), Champaneria R, Daniels JP, et al. Hysterectomy, endometrial ablation and Mirena ${ }^{\circledR}$ for heavy menstrual bleeding: a systematic review of clinical effectiveness and cost-effectiveness analysis. Health Technol Assess. 2011;15(19):iii-xvi, 1-252.

5. Davis E, Sparzak PB. Abnormal uterine bleeding (dysfunctional uterine bleeding). In: StatPearls. Treasure Island: StatPearls Publishing; 2020.

6. Dickersin K, Munro MG, Clark M, Langenberg P, Scherer R, Frick K, et al: Surgical Treatments Outcomes Project for Dysfunctional Uterine Bleeding (STOP-DUB) Research Group. Hysterectomy compared with endometria ablation for dysfunctional uterine bleeding: a randomized controlled trial. Obstet Gynecol. 2007;110(6):1279-89.

7. Munro MG, Dickersin K, Clark MA, Langenberg P, Scherer RW, Frick KD; Surgical Treatments Outcomes Project for Dysfunctional Uterine Bleeding Group. The Surgical Treatments Outcomes Project for Dysfunctional Uterine Bleeding: summary of an Agency for Health Research and Quality-sponsored randomized trial of endometrial ablation versus hysterectomy for women with heavy menstrual bleeding. Menopause. 2011;18(4):445-52.

8. van der Meij E, Emanuel MH. Hysterectomy for heavy menstrual bleeding. Women's Health (Lond). 2016;12(1):63-9.

9. Bongers MY. Hysteroscopy and heavy menstrual bleeding (to cover TCRE and second-generation endometrial ablation). Best Pract Res Clin Obstet Gynaecol. 2015;29(7):930-9.

10. Leathersich SJ, McGurgan PM. Endometrial resection and global ablation in the normal uterus. Best Pract Res Clin Obstet Gynaecol. 2018;46:84-98.

11. Jain $P$, Rajaram $S$, Gupta $B$, Goel $N$, Srivastava $H$. Randomized controlled tria of thermal balloon ablation versus vaginal hysterectomy for leiomyoma-induced heavy menstrual bleeding. Int J Gynaecol Obstet. 2016;135(2):140-4

12. Sesti F, Ruggeri $\bigvee$, Pietropolli $A$, Piancatelli R, Piccione E. Thermal balloon ablation versus laparoscopic supracervical hysterectomy for the surgical treatment of heavy menstrual bleeding: a randomized study. | Obstet Gynaecol Res. 2011;37(11):1650-7.

13. Zupi E, Centini G, Lazzeri L, Finco A, Exacoustos C, Afors K, et al. Hysteroscopic endometrial resection versus laparoscopic supracervical hysterectomy for abnormal uterine bleeding: long-term follow-up of a randomized trial. . Minim Invasive Gynecol. 2015;22(5):841-5.

14. Bonafede MM, Miller ID, Lukes A, Meyer NM, Lenhart GM. Comparison of direct and indirect costs of abnormal uterine bleeding treatment with global endometrial ablation and hysterectomy. J Comp Eff Res. 2015;4(2):115-22.

15. Cooper K, Lee A, Chien P, Raja E, Timmaraju V, Bhattacharya S. Outcomes following hysterectomy or endometrial ablation for heavy menstrual bleeding: retrospective analysis of hospital episode statistics in Scotland. BJOG. 2011;118(10):1171-9.

16. Vitale SG, Ferrero S, Ciebiera M, Barra F, Török P, Tesarik J, et al. Hysteroscopic endometrial resection vs. hysterectomy for abnormal uterine bleeding: impact on quality of life and sexuality. Evidence from a systematic review of randomized controlled trials. Curr Opin Obstet Gynecol. 2020;32(2):159-65.

17. Cheng HY, Lin BL, Tseng JY, Ueno K, Nakada S. Clinical application of Lin's biopsy grasper for intrauterine targeted biopsy and polypectomy during office hysteroscopy. Taiwan J Obstet Gynecol. 2018;57(3):379-82.

18. Vitale SG. The biopsy snake grasper Sec. VITALE: a new tool for office hysteroscopy. | Minim Invasive Gynecol. 2019;S1553-4650(19):31362-7. 